\title{
Performance of Wheat (Triticum aestivum L.) under Tillage Practices and Water Regime on Water Productivity and Economics
}

\author{
Vineet Kumar $^{1 *}$, R.K. Naresh ${ }^{1}$, Vipin Kumar Sagar ${ }^{1}$ and Praveen Kumar Sagar ${ }^{2}$ \\ ${ }^{1}$ Department of Agronomy; Sardar Vallabhbhai Patel University of Agriculture \& \\ Technology, Meerut-250110, U.P., India \\ ${ }^{2}$ Department of Agronomy, K.D. College Simbholi, Chaudary Charan Singh University, \\ Meerut-245207, U.P., India \\ *Corresponding author
}

A B S T R A C T

A field experiment was conducted during 2013-14 and 2014-15 at Crop Research Centre of Sardar Vallabhbhai Patel University of Agriculture and Technology, Meerut-250110 (U.P.).The objective of our study was to establish an understanding of how nutrient uptake

Keywords

Tillage, Raised bed, IW/CPE, WUE, Water productivity, B: C, Wheat

Article Info

Accepted: 26 February 2018 Available Online: 10 March 2018 and wheat yield can be improved and how land configuration and tillage practices can be modified to be more efficient in water use through layering of precision- conservation crop management techniques. The experiment consisting of five tillage practices $T_{1^{-}}$Wide raised bed; $\mathrm{T}_{2}$ - Narrow raised bed; $\mathrm{T}_{3}$ - Conventional tillage; $\mathrm{T}_{4}$ - Reduced tillage; $\mathrm{T}_{5^{-}}$Zero tillage and three treatments viz., $\mathrm{I}_{1}-\mathrm{IW} / \mathrm{CPE} 0.45$; $\mathrm{I}_{2}-\mathrm{IW} / \mathrm{CPE} 0.60 ; \mathrm{I}_{3}-\mathrm{IW} / \mathrm{CPE}$ 0.75; was laid out in split plot design with three replications. The maximum net profit among tillage practices Rs 64301.7 and Rs $60904.3 \mathrm{ha}^{-1}$ with $\mathrm{B}$ : C ratio 2.24 and 2.00 were recorded in wide raised beds plots. The wide raised beds plots increased the water use efficiency of 15.12 and $15.78 \mathrm{~kg}$ grain $\mathrm{ha}^{-1} \mathrm{~mm}$. The per cent increased in water use efficiency under wide raised beds over conventional tillage was 38.67 and $39.23 \%$. Among water regime highest net profit (Rs 65256.4 and Rs 61976.7 ha ${ }^{-1}$ ) with B: C ratio of 2.24 and 2.04 was recorded with the application of three irrigations at 22, 65 and 105 DAS in wheat crop. The wide raised beds plots increased the water use efficiency of 15.12 and $15.78 \mathrm{~kg} \mathrm{ha}^{-3}$ and water productivity (1.28 and $\left.1.18 \mathrm{~kg} \mathrm{ha}^{-3}\right)$. The per cent increased in water use efficiency under wide raised beds over conventional tillage was 38.67 and $39.23 \%$ during 2013-14 and 2014-15, respectively.

\section{Introduction}

Wheat (Triticum aestivum L. emend. Fiori \& Paol.) is very important and remunerative rabi crop of North India. It is the second most important cereal crop after rice, grown under diverse agro-climatic conditions on $28 \mathrm{~m}$ ha area in India with a production of $84 \mathrm{~m}$ tonnes
(Economic Survey, 2010-11). The North West India, which serves as India's food basket, may become food-insecure in the near future. Therefore, there is an urgent need to develop innovative alternative strategies for the future transformation of the irrigated wheat system toward improved practices, ones that (1) are more resource-use-efficient, (2) lead to food 
security, and (3) are economically sustainable. Conservation agriculture (CA)-based resource-conserving technologies (RCTs) include many new technologies (more efficient implements; reduced or minimal tillage; soil, water, and crop management practices) that are more efficient, use less inputs, improve production and income, and attempt to overcome emerging problems (Gupta and Sayre, 2007). Alternative methods have been proven effective to sustain soil health and reduce water demand in the wheat crop in on-station trials in different agroecological regions by many scientists. But the applications of these new tillage and crop establishment methods need to be tested on a wider scale for water, labour, and energy efficiency on farm managed trials (Naresh $e t$ al., 2011). There is a need to develop technologies and management practices that can simultaneously enhance production, preserve the natural resource base, and reduce poverty. In today's so stem, it is necessary to reduce the cost of production and to increase the productivity of wheat in order to compete in the international market. It has been well established that the zero tillage system reduces the cost of cultivation due to single tractor pass (Chauhan et al., 2000). Water is an important input for realizing high wheat productivity; however, it is becoming the most limiting factor for crop production in most of the north western parts of India (Hira, 2009). As water for irrigation is a scarce resource, its optimum use optimization is fundamental to water resource use. It permits better utilization of all other production factors and thus leads to increased yields per unit area and time. Efficient water management requires a thorough study of plant water relationship, climate, agronomic practices and economic assessment. In cultivation of high yielding wheat varieties, irrigation assumes greater importance because during growing season of crop (October to April) weather remains relatively dry. The judicial application of water calls for immediate attention and this is possible only by following some scientific basis for water application to the crop. Various agronomic practices have been developed to conserve rain or irrigation water. Proper utilization of conserved moisture is an effort towards increasing crop yield per unit amount of water used. Most of these practices increase the duration of moisture availability through an increase in the amount of available moisture in the soil. To increase the duration of moisture availability with the existing available moisture, the losses of it from plants (transpiration) and soil (evaporation) have to be reduced. Proper scheduling of irrigation (amount and timing) crops is an important component of water saving techniques. There are numerous ways to schedule irrigations and estimate the required depth of water application (Campbell and Cambell, 1982; Priharand Khepar, 1997). All irrigation scheduling methods consist of monitoring indicators that determine the need for irrigation (Prihar et al., 1974). Suggested a simple approach based on meteorological parameters to schedule irrigation of crops based on the ratio between fixed depth (75 $\mathrm{mm}$ ) of irrigation water (IW) and net cumulative pan evaporation since previous irrigation (PAN-E minus rainfall).Being the prime natural resource for assured crop production, water has to be used judiciously and in a scientific manner.

\section{Materials and Methods}

The experiment was conducted at Crop Research Centre (CRC) of the University situated in Indo-Genetic plains of western Uttar Pradesh in Western Plains Zone. It's geographically located at $29^{\circ} 05^{\prime} 19^{\prime \prime} \mathrm{N}$ latitude, $77^{\circ} 41^{\prime} 50^{\prime \prime} \mathrm{E}$ longitudes and at an elevation of 237 metres above the sea level during rabi2013-14 and 2014-15at a same site in both the years. The climate of this region is semi-arid and sub-tropical with extremes hot 
weather in summer and cold weather in winter season. There is gradual decrease in mean daily temperature in January reaching as low as $5.6^{\circ} \mathrm{C}$ and further a gradual increase is registered reaching as high as $36.6{ }^{0} \mathrm{C}$ in months of April. Occasionally, frost does occur during the months of December and January. The maximum temperature was highest in fourth week of April during both the years. Rainfall was occurred $177.0 \mathrm{~mm}$ and $203.3 \mathrm{~mm}$ during crop period in 2013-14 and 2014-15. The mean weekly weather data for the crop period of 2013-14 and 2014-15. The soil was sandy loam with $\mathrm{pH} 8.0$ and 7.9 (1:2.5 soil to water). The top soil of the experimental site was sandy loam overlying silty clay, with an abrupt change to sandy loam at about $90 \mathrm{~cm}$. Bulk density was 1.51 and $1.56 \mathrm{~g} / \mathrm{cm}$ in the top-soil. Organic carbon 0.50 and $0.51 \%$, available N-242.7 and $244.5 \mathrm{~kg} \mathrm{ha}^{-1}$ available P-12.0 and $12.3 \mathrm{~kg} \mathrm{ha}^{-1}$ available $\mathrm{K} 201.3$ and $202.2 \mathrm{~kg} \mathrm{ha}^{-1}$ at the start of the experiment in 0 to $15 \mathrm{~cm}$ soil layer during 2013-14 and 2014-15, respectively. The treatments consists of five tillage practices $\left(T_{1}\right.$, Wide raised beds, $T_{2}$, Narrow raised beds, $\mathrm{T}_{3}$, Conventional tillage, $\mathrm{T}_{4}$, Reduced tillage, $\mathrm{T}_{5}$, Zero tillage) and three irrigation schedules $\left(\mathrm{I}_{1}-\mathrm{IW} / \mathrm{CPE} 0.45, \mathrm{I}_{2}\right.$ IW/CPE 0.60, I $\mathrm{I}_{3}$ - IW/CPE 0.75). The study was made in split plot design with three replications. In FIRBS, $15 \mathrm{~cm}$ high and $45 \mathrm{~cm}$ broad bed with a furrow width of $25 \mathrm{~cm}$ between the beds was prepared with planting three rows of wheat in rows $15 \mathrm{~cm}$ apart (Jat et al., 2005). Half dose of $\mathrm{N}$ and full dose of $\mathrm{P}$ and $\mathrm{K}$ through urea, single super phosphate and muriate of potash, respectively, were applied at sowing and remaining $\mathrm{N}$ was applied after first irrigation. Wheat DBW-17 was sown on 15 November and 22 November, in 2013 and 2014 and harvested on 15 April and 20 April, in 2014 and 2015, respectively. Other management practices were adopted as per recommendations of the crop under irrigated conditions. The nutrient uptake by the crops was obtained as product of nutrient concentration and yield. Two years data was pooled and statistically analyzed. Soil moisture content was measured at seeding, and before and after each irrigation on the top of the ridge and furrow in furrow irrigated raised bed planting system, between the 2 rows in flat planting by gravimetric method. Water saving (WS) was calculated as:

$\mathrm{WS}=\left(\mathrm{Q}_{\mathrm{F}}-\mathrm{Q}_{\mathrm{B}}\right) / \mathrm{Q}_{\mathrm{F}} \times 100$,

Where $\mathrm{Q}_{\mathrm{F}}$ and $\mathrm{Q}_{\mathrm{B}}$ are quantity of water applied in flat planting and furrow irrigated raised bed planting system, respectively. The soil moisture data will be utilized to calculate the consumptive use.

\section{Results and Discussion}

\section{Soil moisture studies}

\section{Moisture extraction pattern}

The data on soil profile moisture extraction pattern of wheat crop under different irrigation levels and tillage practices treatments is shown in Table $1(\mathrm{a}, \mathrm{b}$ and $\mathrm{c})$. The soil profile was divided in four layers $(0-15,15-30,30-60$ and $60-90 \mathrm{~cm}$ ) and the maximum amount of water was extracted (absorbed) from 30-60 cm layer followed by $0-15 \mathrm{~cm}, 15-30 \mathrm{~cm}$, and minimum from $60-90 \mathrm{~cm}$ during both the years of study. The moisture extraction from the surface layer $(0-15 \mathrm{~cm})$ was increased slightly with increase in irrigation frequency during both the years of study.

Similarly, the moisture extraction was decreased slightly with increase in profile depth and irrigation frequency during both the years. The raised beds/zero till plots consumed more moisture from the deeper profile layer than conventional tillage practice and viceversa during both the years of study (Kingra and Mahey, 2013; Singh et al., 2015). 


\section{Soil moisture content studies}

The effect of tillage practices under three water regimes on average profile soil moisture content Table 1 ( $\mathrm{a}, \mathrm{b}$ and $\mathrm{c})$. In general, the profile moisture content was highest at the time of sowing (21\%) and it was lowest at the time of crop maturity in all the treatments during both the years of study. The increases in profile moisture content are visible from the peaks under different water regimes were because of application of irrigation as per treatments. The moisture content of conventional tilled plots $\left(\mathrm{T}_{3}\right)$ was always lower than furrow irrigated raised beds plots $\left(T_{1} \& T_{2}\right)$ during both the years of study except in the peaks where the moisture content in the profile was always same due to recharging of profile by application of irrigation. In between the season the lowest soil moisture content in conventional till crop and furrow irrigated raised beds plots was 74 DAS (16.1\%) in $\mathrm{I}_{1}$, 104 DAS (16.2\%) in $\mathrm{I}_{2}$ and 90 DAS (15\%) in $\mathrm{I}_{3}$ during 2013-14, whereas, the lowest soil profile moisture content during 2014-15 in $\mathrm{I}_{1}$ was recorded at 97 DAS (17\%), in $\mathrm{I}_{2} 60$ DAS $(17.5 \%)$ and in $\mathrm{I}_{3} 97$ DAS (16.9\%). The conventional till crop under all the water regimes kept the average profile soil moisture content $1.5 \%$ lower than furrow irrigated raised beds plots throughout the crop season except after recharging the soil profile either by application of irrigation as per treatment or by rainfall.

\section{Seasonal water use}

The data on crop seasonal water use under different irrigation and tillage practices crop during 2013-14 and 2014-15 is shown in Table 2. The seasonal water use increase with advancement of crop season in all the treatment during both the years of study. The highest water use was observed in $\mathrm{I}_{3}$ followed by $\mathrm{I}_{2}$ and it was lowest in $\mathrm{I}_{1}$ during both years. Among the tillage practices raised beds/zero till plots treatment the crop consumed markedly higher water than conventional tillage plots during the crop season. The crop consumed highest amount of water between 90-120 days $(9.8$ and $8.1 \mathrm{~cm}$ ) during both the years of study. The lowest amount of water was consumed during first 30 days (3.2 and $3.1 \mathrm{~cm}$ ) during both the years the water use on per day basis was maximum between 120 and the maturity of the crop.These finding was supported by Saren et al., (2004).

\section{Water use and water use efficiency}

The crop water use during 2014-15 was slightly higher than 2013-14 (Table 2). The crop water use increased with increasing irrigation during both the years.

The crop water use increased markedly in conventional till plots $\left(\mathrm{T}_{3}\right)$ than raised beds crop plots $\left(\mathrm{T}_{1}\right)$ during both the years. The water use efficiency increased with the increased level of irrigation frequency during experimentation. The WUE was remarkably low in conventional till plots crop $\left(\mathrm{T}_{3}\right)$ than raised beds crop plots $\left(T_{1}\right.$ and $\left.T_{2}\right)$ during both the years of study (Singh and katiyar 2014 and Singh et al., 2015).

\section{Water productivity}

The average water productivity (Table 3 ) was higher in the bed planting $\mathrm{T}_{1}(1.28$ and $1.18 \mathrm{~kg}$ $\mathrm{ha}^{-3}$ ) followed by conventional $(0.92$ and 0.86 $\mathrm{kg} \mathrm{ha}^{-3}$ ) and zero tillage (1.02 and $0.94 \mathrm{~kg}$ $\mathrm{ha}^{-3}$ ) might be due to the less consumptive use of water resulted in higher) reported higher in bed planting as compared to conventional planting with $I_{3}$. The maximum water productivity was observed where irrigation was applied at $\left(\mathrm{I}_{3}\right) \mathrm{IW}$ : CPE ratio of $0.75(0.91$ and $\left.0.83 \mathrm{~kg} \mathrm{~kg} \mathrm{ha}^{-3}\right)$ than $\left(\mathrm{I}_{2}\right) \mathrm{IW}$ : CPE ratio of 0.60 (1.01 and $\left.0.92 \mathrm{~kg} \mathrm{~kg} \mathrm{ha}^{-3}\right)$ and $\left(\mathrm{I}_{1}\right) 0.45$ IW: CPE ratio (0.91 and $\left.0.83 \mathrm{~kg} \mathrm{~kg} \mathrm{ha}{ }^{-3}\right)$ could be due to the prolonged availability of soil water content resulted in higher grain yield. 
Table.1 (a) The moisture depletion pattern of $\mathrm{I}_{1}$ different soil layers as influenced by tillage practices and water regime

\begin{tabular}{|c|c|c|c|c|c|c|c|c|c|c|}
\hline \multirow[t]{3}{*}{ Treatment } & \multicolumn{10}{|c|}{$\mathrm{I}_{1} 0.45 \mathrm{IW} / \mathrm{CPE}$ (water regime) } \\
\hline & \multicolumn{2}{|c|}{$0-15 \mathrm{~cm}$} & \multicolumn{2}{|c|}{$15-30 \mathrm{~cm}$} & \multicolumn{2}{|c|}{$30-60 \mathrm{~cm}$} & \multicolumn{2}{|c|}{$60-90 \mathrm{~cm}$} & \multicolumn{2}{|c|}{$\begin{array}{l}\text { Total moisture } \\
\text { depletion }(\mathrm{cm})\end{array}$} \\
\hline & $\begin{array}{c}2013- \\
14\end{array}$ & $\begin{array}{c}2014- \\
15\end{array}$ & $\begin{array}{c}2013- \\
14\end{array}$ & 2014-5 & $\begin{array}{c}2013- \\
14\end{array}$ & $\begin{array}{l}2014- \\
15\end{array}$ & $\begin{array}{c}2013- \\
14\end{array}$ & $\begin{array}{l}2014- \\
15\end{array}$ & $\begin{array}{c}2013- \\
14\end{array}$ & $\begin{array}{c}2014- \\
15\end{array}$ \\
\hline \multicolumn{11}{|c|}{ Tillage practices } \\
\hline $\mathrm{T}_{1}$ & 2.52 & 2.56 & 4.20 & 4.22 & 3.58 & 3.55 & 6.54 & 6.55 & 16.84 & 16.88 \\
\hline $\mathbf{T}_{2}$ & 3.17 & 3.15 & 4.80 & 4.81 & 4.66 & 4.64 & 7.20 & 7.22 & 19.83 & 19.82 \\
\hline $\mathbf{T}_{3}$ & 3.78 & 3.80 & 7.35 & 7.33 & 5.26 & 5.25 & 7.73 & 7.73 & 24.12 & 24.11 \\
\hline $\mathbf{T}_{4}$ & 3.76 & 3.76 & 7.09 & 7.10 & 4.97 & 4.92 & 6.92 & 6.90 & 22.74 & 22.68 \\
\hline $\mathbf{T}_{5}$ & 3.53 & 3.55 & 6.72 & 6.73 & 4.77 & 4.76 & 7.45 & 7.48 & 22.47 & 22.52 \\
\hline
\end{tabular}

Table.1 (b) The moisture depletion pattern of $\mathrm{I}_{2}$ from different soil layers as influenced by tillage practices and water regime

\begin{tabular}{|c|c|c|c|c|c|c|c|c|c|c|}
\hline \multirow[t]{3}{*}{ Treatment } & \multicolumn{10}{|c|}{$\mathrm{I}_{2} \mathbf{0 . 6 0 I W / C P E}$ (water regime) } \\
\hline & \multicolumn{2}{|c|}{$0-15 \mathrm{~cm}$} & \multicolumn{2}{|c|}{$15-30 \mathrm{~cm}$} & \multicolumn{2}{|c|}{$30-60 \mathrm{~cm}$} & \multicolumn{2}{|c|}{$60-90 \mathrm{~cm}$} & \multicolumn{2}{|c|}{$\begin{array}{l}\text { Total moisture } \\
\text { depletion }(\mathrm{cm})\end{array}$} \\
\hline & $\begin{array}{c}2013- \\
14\end{array}$ & 2014-15 & $\begin{array}{c}2013- \\
14\end{array}$ & $\begin{array}{c}2014- \\
15\end{array}$ & $\begin{array}{c}2013- \\
14\end{array}$ & $\begin{array}{c}2014- \\
15\end{array}$ & $\begin{array}{c}2013- \\
14\end{array}$ & $\begin{array}{c}\text { 2014- } \\
15\end{array}$ & $\begin{array}{c}2013- \\
14\end{array}$ & $\begin{array}{c}2014- \\
15\end{array}$ \\
\hline \multicolumn{11}{|c|}{ Tillage practices } \\
\hline $\mathrm{T}_{1}$ & 2.20 & 2.22 & 3.45 & 4.45 & 3.75 & 3.77 & 1.10 & 1.11 & 10.50 & 11.55 \\
\hline $\mathbf{T}_{2}$ & 3.03 & 3.08 & 5.13 & 5.15 & 4.63 & 4.63 & 2.59 & 2.61 & 15.38 & 15.47 \\
\hline $\mathbf{T}_{3}$ & 3.76 & 3.78 & 7.50 & 7.48 & 5.40 & 5.40 & 3.79 & 3.78 & 20.45 & 20.44 \\
\hline $\mathbf{T}_{4}$ & 3.83 & 3.83 & 7.29 & 7.26 & 4.82 & 7.80 & 3.49 & 3.48 & 19.43 & 22.37 \\
\hline $\mathbf{T}_{5}$ & 3.43 & 3.41 & 7.03 & 7.05 & 4.81 & 4.78 & 3.01 & 3.00 & 18.28 & 18.24 \\
\hline
\end{tabular}

Table.1 (c) The moisture depletion pattern of $\mathrm{I}_{3}$ from different soil layers as influenced by tillage practices and water regime

\begin{tabular}{|c|c|c|c|c|c|c|c|c|c|c|}
\hline \multirow[t]{3}{*}{ Treatment } & \multicolumn{10}{|c|}{ I 0.75 IW/CPE(water regime) } \\
\hline & \multicolumn{2}{|c|}{$0-15 \mathrm{~cm}$} & \multicolumn{2}{|c|}{$15-30 \mathrm{~cm}$} & \multicolumn{2}{|c|}{$30-60 \mathrm{~cm}$} & \multicolumn{2}{|c|}{$60-90 \mathrm{~cm}$} & \multicolumn{2}{|c|}{$\begin{array}{l}\text { Total moisture } \\
\text { depletion }(\mathrm{cm})\end{array}$} \\
\hline & $\begin{array}{c}2013- \\
14\end{array}$ & $\begin{array}{c}2014- \\
15\end{array}$ & $\begin{array}{c}2013- \\
14\end{array}$ & $\begin{array}{c}2014- \\
15\end{array}$ & $\begin{array}{c}2013- \\
14\end{array}$ & $\begin{array}{c}2014- \\
15\end{array}$ & $\begin{array}{c}2013- \\
14\end{array}$ & $\begin{array}{l}2014- \\
15\end{array}$ & $\begin{array}{c}2013- \\
14\end{array}$ & $\begin{array}{c}2014- \\
15\end{array}$ \\
\hline \multicolumn{11}{|c|}{ Tillage practices } \\
\hline $\mathrm{T}_{1}$ & 2.46 & 2.45 & 3.48 & 3.46 & 2.56 & 2.58 & 0.67 & 1.01 & 9.17 & 9.50 \\
\hline $\mathbf{T}_{2}$ & 3.74 & 3.75 & 4.74 & 4.75 & 3.16 & 3.15 & 1.49 & 1.50 & 13.13 & 13.15 \\
\hline $\mathbf{T}_{3}$ & 3.82 & 3.80 & 6.77 & 6.79 & 4.22 & 4.20 & 2.54 & 2.52 & 17.35 & 17.31 \\
\hline $\mathbf{T}_{4}$ & 3.79 & 3.80 & 6.13 & 6.12 & 3.71 & 3.73 & 2.13 & 2.13 & 15.76 & 15.78 \\
\hline $\mathbf{T}_{5}$ & 3.19 & 3.18 & 5.44 & 5.44 & 3.00 & 2.98 & 2.00 & 1.97 & 13.63 & 13.57 \\
\hline
\end{tabular}


Table.2 Seasonal consumptive use and water use efficiency as influenced by tillage practices and water regime

\begin{tabular}{|c|c|c|c|c|c|c|c|c|c|c|c|c|c|c|c|c|}
\hline \multirow[t]{5}{*}{ Treatment } & \multicolumn{16}{|c|}{ Water regime } \\
\hline & \multicolumn{7}{|c|}{ Consumptive use (cm) } & \multicolumn{9}{|c|}{ 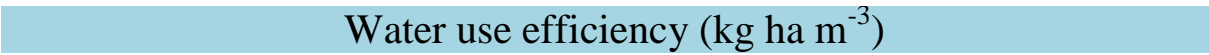 } \\
\hline & \multicolumn{2}{|c|}{$I_{1}(0.45)$} & \multicolumn{2}{|c|}{$\mathbf{I}_{2}(\mathbf{0 . 6 0 )}$} & \multicolumn{2}{|c|}{$\mathbf{I}_{3}(\mathbf{0 . 7 5})$} & \multicolumn{2}{|c|}{ Mean } & \multicolumn{2}{|c|}{$I_{1}(0.45)$} & \multicolumn{2}{|c|}{$\mathbf{I}_{2}(\mathbf{0 . 6 0 )}$} & \multicolumn{2}{|c|}{$I_{3}(0.75)$} & \multicolumn{2}{|c|}{ Mean } \\
\hline & 2013 & 2014 & 2013 & 2014 & 2013 & 2014 & 2013 & 2014 & 2013 & 2014 & 2013 & 2014 & 2013 & 2014 & 2013 & 2014 \\
\hline & -14 & -15 & -14 & -15 & -14 & -15 & -14 & -15 & -14 & -15 & -14 & -15 & -14 & -15 & -14 & -15 \\
\hline \multicolumn{17}{|c|}{ Tillage practices } \\
\hline $\mathbf{T}_{1}$ & 36.05 & 38.90 & 29.51 & 32.32 & 28.18 & 31.33 & 31.25 & 34.18 & 1.17 & 1.06 & 1.67 & 1.50 & 1.97 & 1.74 & 1.57 & 1.41 \\
\hline $\mathbf{T}_{2}$ & 38.84 & 41.64 & 34.19 & 37.01 & 31.94 & 34.79 & 34.99 & 37.82 & 1.10 & 1.00 & 1.36 & 1.24 & 1.62 & 1.47 & 1.34 & 1.22 \\
\hline $\mathbf{T}_{3}$ & 42.92 & 45.51 & 39.06 & 41.89 & 35.96 & 38.75 & 39.31 & 42.05 & 0.85 & 0.78 & 1.11 & 1.02 & 1.31 & 1.19 & 1.08 & 0.99 \\
\hline$T_{4}$ & 41.55 & 44.10 & 38.04 & 40.87 & 34.37 & 36.99 & 37.99 & 40.65 & 0.95 & 0.86 & 1.21 & 1.08 & 1.40 & 1.25 & 1.17 & 1.05 \\
\hline $\mathbf{T}_{5}$ & 41.09 & 43.98 & 36.93 & 39.77 & 32.04 & 34.82 & 36.69 & 39.52 & 1.07 & 0.97 & 1.18 & 1.07 & 1.49 & 1.34 & 1.23 & 1.11 \\
\hline Mean & 40.09 & 42.82 & 35.55 & 38.37 & 32.50 & 35.34 & 36.05 & 38.84 & 1.02 & 0.93 & 1.29 & 1.16 & 1.54 & 1.38 & 1.26 & 1.15 \\
\hline
\end{tabular}

Table.3 Water use pattern and water productivity of wheat as influenced by tillage practices and water regime

\begin{tabular}{|c|c|c|c|c|c|c|c|c|c|c|c|c|}
\hline \multirow[t]{4}{*}{ Treatment } & \multicolumn{12}{|c|}{ Water regime } \\
\hline & \multicolumn{4}{|c|}{$\mathrm{I}_{1}(0.45$ IW/CPE) } & \multicolumn{4}{|c|}{$\mathrm{I}_{2}(0.60 \mathrm{IW} / \mathrm{CPE})$} & \multicolumn{4}{|c|}{$\mathrm{I}_{3}(\mathbf{0 . 7 5 I W / C P E})$} \\
\hline & \multicolumn{2}{|c|}{ 2013-14 } & \multicolumn{2}{|c|}{ 2014-15 } & \multicolumn{2}{|c|}{ 2013-14 } & \multicolumn{2}{|c|}{ 2014-15 } & \multicolumn{2}{|c|}{ 2013-14 } & \multicolumn{2}{|c|}{ 2014-15 } \\
\hline & $\begin{array}{c}\text { Total } \\
\text { water } \\
\text { use } \\
(\mathrm{cm})\end{array}$ & $\begin{array}{c}\text { Water } \\
\text { producti } \\
\text { vity } \\
\left(\mathrm{kg} \mathrm{m}^{-3}\right)\end{array}$ & $\begin{array}{c}\text { Total } \\
\text { water } \\
\text { use } \\
(\mathrm{cm})\end{array}$ & $\begin{array}{c}\text { Water } \\
\text { producti } \\
\text { vity } \\
\left(\mathrm{kg} \mathrm{m}^{-3}\right)\end{array}$ & $\begin{array}{c}\text { Total } \\
\text { water } \\
\text { use } \\
(\mathrm{cm})\end{array}$ & $\begin{array}{c}\text { Water } \\
\text { producti } \\
\text { vity } \\
\left(\mathrm{kg} \mathrm{m}^{-3}\right)\end{array}$ & $\begin{array}{c}\text { Total } \\
\text { water } \\
\text { use } \\
(\mathrm{cm})\end{array}$ & $\begin{array}{c}\text { Water } \\
\text { producti } \\
\text { vity } \\
\left(\mathrm{kg} \mathrm{m}^{-3}\right)\end{array}$ & $\begin{array}{c}\text { Total } \\
\text { water } \\
\text { use } \\
(\mathrm{cm})\end{array}$ & $\begin{array}{c}\text { Water } \\
\text { producti } \\
\text { vity } \\
\left(\mathrm{kg} \mathrm{m}^{-3}\right)\end{array}$ & $\begin{array}{c}\text { Total } \\
\text { water } \\
\text { use } \\
(\mathrm{cm})\end{array}$ & $\begin{array}{c}\text { Water } \\
\text { producti } \\
\text { vity } \\
\left(\mathrm{kg} \mathrm{m}^{-3}\right)\end{array}$ \\
\hline \multicolumn{13}{|c|}{ Tillage practices } \\
\hline $\mathrm{T}_{1}$ & 41.05 & 1.03 & 43.90 & 0.94 & 39.51 & 1.25 & 42.32 & 1.15 & 43.18 & 1.28 & 46.33 & 1.18 \\
\hline $\mathbf{T}_{2}$ & 43.84 & 0.97 & 46.64 & 0.89 & 44.19 & 1.05 & 47.01 & 0.97 & 46.94 & 1.10 & 49.79 & 1.02 \\
\hline $\mathbf{T}_{3}$ & 47.92 & 0.76 & 50.51 & 0.71 & 49.06 & 0.89 & 51.89 & 0.82 & 50.96 & 0.92 & 53.75 & 0.86 \\
\hline$T_{4}$ & 46.55 & 0.85 & 49.10 & 0.77 & 48.04 & 0.95 & 50.87 & 0.87 & 49.37 & 0.97 & 51.99 & 0.89 \\
\hline $\mathbf{T}_{5}$ & 46.09 & 0.95 & 48.98 & 0.87 & 46.93 & 0.93 & 49.77 & 0.85 & 47.04 & 1.02 & 49.82 & 0.94 \\
\hline Mean & 45.09 & 0.91 & 47.82 & 0.83 & 45.55 & 1.01 & 48.37 & 0.92 & 47.50 & 1.05 & 50.34 & 0.97 \\
\hline
\end{tabular}

Total water used by the crop includes Soil moisture contribution, moisture depletion pattern, effective rainfall and irrigation applied 
Table.4 Effect of tillage practices and water regime on grain, straw, 1000 grain weight and harvest index

\begin{tabular}{|c|c|c|c|c|c|c|c|c|}
\hline \multirow[t]{2}{*}{ Treatment } & \multicolumn{2}{|c|}{ Grain yield $\left(q\right.$ ha $\left.^{-1}\right)$} & \multicolumn{2}{|c|}{ Straw yield $\left(q\right.$ ha $\left.^{-1}\right)$} & \multicolumn{2}{|c|}{1000 grain weight (g) } & \multicolumn{2}{|c|}{ Harvest index (\%) } \\
\hline & 2013-14 & 2014-15 & 2013-14 & 2014-15 & 2013-14 & 2014-15 & 2013-14 & 2014-15 \\
\hline \multicolumn{9}{|l|}{ Tillage practices } \\
\hline $\mathrm{T}_{1}$ & 48.96 & 48.19 & 62.78 & 61.55 & 46.7 & 45.7 & 43.81 & 43.83 \\
\hline $\mathbf{T}_{2}$ & 47.01 & 46.17 & 58.51 & 57.55 & 46.3 & 45.6 & 44.54 & 44.05 \\
\hline$T_{3}$ & 42.34 & 41.45 & 51.47 & 50.57 & 44.0 & 43.2 & 45.14 & 44.59 \\
\hline $\mathbf{T}_{4}$ & 44.54 & 42.81 & 53.17 & 51.95 & 44.2 & 43.6 & 42.97 & 44.11 \\
\hline$T_{5}$ & 45.07 & 43.90 & 59.12 & 56.81 & 45.2 & 44.7 & 45.88 & 45.77 \\
\hline $\operatorname{SEm}( \pm)$ & 0.77 & 0.82 & 1.33 & 1.67 & 0.38 & 0.35 & - & - \\
\hline C.D. $(P=0.05)$ & 2.51 & 2.69 & 4.35 & 4.79 & 1.25 & 1.13 & - & - \\
\hline \multicolumn{9}{|l|}{ Water regime } \\
\hline$I_{1}$ & 40.95 & 39.87 & 51.19 & 49.84 & 44.3 & 43.8 & 44.46 & 44.29 \\
\hline $\mathbf{I}_{2}$ & 45.78 & 44.70 & 57.31 & 55.96 & 45.1 & 44.5 & 44.46 & 44.65 \\
\hline $\mathbf{I}_{3}$ & 50.02 & 48.94 & 62.54 & 61.26 & 46.4 & 45.3 & 44.48 & 44.47 \\
\hline $\operatorname{SEm}( \pm)$ & 0.50 & 0.54 & 0.64 & 0.67 & 0.42 & 0.38 & - & - \\
\hline C.D. $(P=0.05)$ & 1.48 & 1.60 & 1.88 & 1.97 & 1.25 & 1.12 & - & - \\
\hline Interaction I ×T & Sig & Sig & Sig & Sig & NS & NS & - & - \\
\hline
\end{tabular}

Table.5 Effect of tillage practices and water regime on profitability

\begin{tabular}{|c|c|c|c|c|c|c|c|c|}
\hline \multirow[t]{2}{*}{ Treatment } & \multicolumn{2}{|c|}{ Gross income (Rs. ha-1) } & \multicolumn{2}{|c|}{ Cost of cultivation (Rs. ha ${ }^{-1}$ ) } & \multicolumn{2}{|c|}{ Net return $\left(\right.$ Rs. ha $\left.{ }^{-1}\right)$} & \multicolumn{2}{|c|}{ B:C ratio } \\
\hline & 2013-14 & 2014-15 & 2013-14 & 2014-15 & 2013-14 & 2014-15 & 2013-14 & 2014-15 \\
\hline \multicolumn{9}{|c|}{ Tillage practices } \\
\hline $\mathrm{T}_{1}$ & 92961.7 & 91414.3 & 28660.0 & 30510.0 & 64301.7 & 60904.3 & 2.24 & 2.00 \\
\hline $\mathbf{T}_{2}$ & 88642.9 & 87089.7 & 28865.0 & 30815.0 & 59777.9 & 56274.7 & 2.07 & 1.83 \\
\hline$T_{3}$ & 79408.4 & 77802.9 & 29600.0 & 31350.0 & 49808.4 & 46452.9 & 1.68 & 1.48 \\
\hline $\mathbf{T}_{4}$ & 85273.0 & 81956.0 & 28550.0 & 30150.0 & 56723.0 & 51806.0 & 1.99 & 1.72 \\
\hline $\mathrm{T}_{5}$ & 83956.3 & 81832.8 & 26537.0 & 27367.0 & 57419.3 & 54465.8 & 2.16 & 1.99 \\
\hline \multicolumn{9}{|l|}{ Water regime } \\
\hline$I_{1}$ & 77290.5 & 75253.4 & 28372.5 & 29638.5 & 48918.0 & 45614.9 & 1.72 & 1.54 \\
\hline $\mathbf{I}_{2}$ & 86432.8 & 84395.7 & 28779.0 & 30045.0 & 57653.8 & 54350.7 & 2.00 & 1.81 \\
\hline $\mathbf{I}_{3}$ & 94421.9 & 92408.2 & 29165.5 & 30431.5 & 65256.4 & 61976.7 & 2.24 & 2.04 \\
\hline
\end{tabular}

Bandyopadhyay (1997) reported that highest was obtained with IW: CPE ratio of 0.75 and decreased with the decrease in frequency of irrigation. Corroborative results in wheat have also been reported by Bandyopadhyay and Mallick (2003) and Kumar and Dhindwal (2009) under shallow water table conditions (Kumar 2010; Singh et al., 2015).

\section{Yield}

Yield is a function of various parameters like crop dry matter accumulation, number of tillers, number of grains spike ${ }^{-1}$ and 1000 grain weight etc. Yield is the most important criteria for evaluating the effects of applied treatments. Crop productivity is the rate at 
which a crop accumulate biomass which depends primarily on the photosynthesis and conversion of light energy into chemical energy by green plants.

The data on crop yield is presented in (Table 4) revealed that crop yield increased with each increment in moisture retention level. The grains yield was higher during 2013-14 and 2014-15. Tillage influenced the grain yield significantly during both the years of study. Wheat sown on wide raised beds $\left(T_{1}\right)$ were recorded the maximum grains yield, straw yield and biological yield and narrow raised beds $\left(\mathrm{T}_{2}\right)$ remained statistically at par with it.

The reduction in grain yield due to more tillage i.e. traditional practices with was 3.06, 2.43 and $3.25 \%$ compared to $\mathrm{T}_{1}$ (wide raised beds), $\mathrm{T}_{2}$ (narrow raised beds) and $\mathrm{T}_{5}$ (zero tillage) practices, respectively. However, wheat sown on wide raised beds registered 11.79 and $11.32 \%$ a significant yield improvement over conventional practices. Significantly higher grain yield straw yield and biological yield was obtained in IW/CPE $0.75\left(\mathrm{I}_{3}\right)$ was treatment which remained statistically at par with $\mathrm{I}_{2}$ treatment. $\mathrm{I}_{1}$ "conventional tillage" treatment recorded minimum grain yield (40.95 and39.87 q ha-1) during the years of study, respectively. Similar reported was Singh and Katiyar (2014) and Sepat et al., (2015). Significantly yield increased with the every successive increase in moisture supply by moisture retention and bed configuration. Similar results of increased yield were also reported by Idnani and Kumar, (2012) and Mishra, et al., (2016). The interaction, tillage $\times$ irrigation water was significant for the grain, straw and biological yield. The magnitude of increase in straw yield due to improvement in moisture supply by tillage modes with wheat sown on raised beds was higher in I3 as compared to other irrigation water.

\section{Profitability}

The economics of wheat crop affected by different tillage practices and water regimes is depicted in (Table 5). The cost of cultivation of wheat crop increase with the increasing of water regimes although the increase was very nominal during both the years. Among the different tillage practices the cost of cultivation was highest in $T_{3}$ conventional tillage followed by reduced tillage/narrow raised beds and it was lowest in zero tillage/wide raised beds practices plots. Among the different tillage practices the highest net profit, gross income, $\mathrm{B}$ : $\mathrm{C}$ ratio was recorded in $\left(T_{1}\right)$ wheat sown on wide raised beds. This may be because of higher water use efficiency than other tillage practices as well as comparatively higher increase in grain yield than in other treatment. The minimum net profit, gross income, B: C was observed in $\left(T_{3}\right)$ conventional tillage treatment during both the years, respectively. From the above results it is clear that increase in irrigation level increased the available moisture in soil, which may be attributed to increase the cost of cultivation, gross income, net profit and $\mathrm{B}$ : $\mathrm{C}$ ratio because of more increase in grain yield and gross income in comparison to increase in cost of cultivation. These results are in conformity with the findings of Kumar et al., (2013); Naresh et al., (2013).

Wide raised beds performed best with highest grain yield followed by narrow raised beds and zero tillage. The increased water regimes increased the growth and yield significantly and it was highest in $I_{3}$ where 3 irrigation were applied at 22, 65 and 105 DAS. The economics of wide raised beds gave maximum return in terms of net profit $\mathrm{Rs}$ $62603 \mathrm{ha}^{-1}$ with a B:C ratio of 2.12 followed by narrow raised beds recommended and zero tillage with $\mathrm{B}: \mathrm{C}$ ratio of 1.95 and 2.07, respectively. The highest net profit of Rs 
65256.40 and Rs 61976.70 ha $^{-1}$ was available with three irrigations applied at 22, 65 and 105 DAS during 2013-14 and 2014-15, respectively. The $\mathrm{B}$ : $\mathrm{C}$ ratio was also highest with three irrigations i.e. 2.24 and 2.04 during 2013-14 and 2014-15. The improved yield, profit and B: $\mathrm{C}$ ratio under conservation tillage over years indicates that consistent build-up of organic matter. Thus for introducing a suitable management practice, it is necessary to now the yield reductions level of plant water deficit in different tillage/water regimes practices. Such information is necessary for crop planning in various agroclimatic conditions both under un-irrigated and irrigated situations. Results of this investigation can be extended to similar soil and climatic condition for wide area.

\section{Acknowledgement}

This study has been executed at the Crop research centre of Sardar Vallabhbhai Patel University of Agriculture and Technology, Meerut, Uttar Pradesh, India under the Department of Agronomy between 2013-14 and 2014-15. I would like to thank the Department of Agronomy for offering me the necessary facilities during this period. Moreover, we would like to express our great respect for the editors and anonymous reviewers to improve the manuscript quality.

\section{References}

Bandyopadhyay, P. K. 1997. Effect of irrigation schedule on evapotranspiration and water use efficiency of winter wheat (Triticum aestivum Indian J. Agron.). 42(1): 90-93.

Bandyopadhyay, P.K and Mallic S. 2003. Actual evapotranspiration and crop coefficients of wheat (Triticum aestivum) under varying moisture levels of humid tropical canal command area. Agricultural Water Management 59: 33-47.

Campbell, G.S. and Cambell, M.D. 1982. Irrigation scheduling using soil moisture measurements: Theory and practice.
Advances in Irrigation. Hillel D (Ed). Academic Press, New York 11: 25-42.

Chauhan, D.S., Sharma, R.K., Tripathi, S.C., Kharub, A.S. and Chhokar, R.S. 2000. Wheat cultivation after rice - a paradigm shift in tillage technology. Indian Farming 50 (6): 21-22.

Gupta, R.K. and Sayre, K. 2007. Conservation agriculture in South Asia. Journal of Agricultural Science 145 (3): 207-214.

Hira, G.S. 2009.Water management in northern states and the food security of India. Journal of Crop Improvement 23: 136-157.

Idnani, L.K. and Kumar, Ashok. 2012. Relative efficiency of different irrigation schedules for conventional, ridge and raised bed seeding of wheat (Triticum aestivum L.). Indian Journal of Agronomy57 (2): 148151.

Jat M L, Singh Samar, Rai H K, Chhokar R S, Sharma S K and Gupta R K. 2005. Furrow irrigated raised bed (FIRB) planting technique for diversification of rice-wheat system in Indo-Gangetic Plains. Japan Association for International Collaboration of Agriculture and Forestry 28 (1): 25-42.

Kingra, P. K. and Mahey, R. K. 2013. Moisture extraction pattern and ET-yield models in wheat under different management practices in central Punjab. Journal of Agrometeorology, 15(1): 51-57.

Kumar, Ashok, Sharma, K. D., Yadav, Ashok. 2010. Enhancing yield and water productivity of wheat (Triticum aestivum) through furrow irrigated raised bed system in the Indo-Gangetic Plains of India. Indian Journal of Agricultural Sciences; 80(3):198-202.

Kumar, M., Das, A., Ramkrushna, G. I., Patel, D. P., Munda, G. C., Naropongla and Buragohain, J. 2013.Effect of nutrient sources and transplanting date on aromatic rice (Oryza sativa) under mid hills of northeastern India. Indian J. Agron., 58 (3): 3226.

Kumar, S and Dhindwal A S. 2009. Water productivity of wheat succeeding mungbean and sorghum in relation to planting techniques and irrigation scheduling. Journal of Water Management 17(2): 1-7. 
Maurya, R. K., Singh, G. R.2008. Effect of crop establishment methods and irrigation schedules on economics of wheat (Triticum aestivum) production, moisture depletion pattern, consumptive use and crop wateruse efficiency. Indian Journal of Agricultural Sciences; 78(10):830-833.

Mishra, Gaurav, Kushwaha, H. S. 2016. Winter wheat yield and soil physical properties responses to different tillage and irrigation. European Journal of Biological Research 2016; 6 (1): 56-63.

Naresh, R.K., Gupta, R.K., Singh, Y.P., Kumar, A., Khilari, K., Singh. P.K. Shahi. U. P., Tomar, S.S. and Yadav, A.K. 2011. Impact of Resource Conservation Technologies for Sustainability of irrigated agriculture in Uttar Pradesh-India. The Journal of Rural and Agricultural Research 11(1): 13-18.

Prihar, S.S. and Khepar, S.D. 1997. Resource management for sustainable intensive agriculture - water. Proc. Third Agricultural Science Congress, National Academy of Agricultural Sciences, Ludhiana, Pp. 91-101.

Prihar, S.S., Gajri, P.R. and Narang, R.S. 1974. Scheduling irrigation to wheat using open pan evaporation. Indian Journal of Agricultural Science 44: 567-71.
Saren, B.K., Dey, S. and Mandal, D. 2004. Effect of irrigation and sulphur on yield attributes, productivity, consumptive use, consumptive use efficiency of wheat (Triticum aestivum L.). Indian Journal of Agricultural Sciences 74 (5): 257-261.

Sepat, Seema, Sharma, A. R., Kumar, Dinesh, and DAS, T. K. 2015 Effect of conservation agriculture practices on productivity and sustainability of pigeonpea (Cajanus cajan)-wheat (Triticum aestivum) cropping system in Indo-Gangetic plains of India. Indian Journal of Agricultural Sciences; 85(2): 212

Singh, Karmal, Dhindwal, A.S., Dhaka, A.K., Sewhag, Meena and Pannu, R.K. 2015. Water use pattern and productivity in bed planted wheat (Triticum aestivum L.) under varying moisture regimes in shallow water table

conditions. Indian Journal of Agricultural Sciences. 85(8): 1080-1084.

Singh, Y. K., Katiyar, T. P. S. 2014. Effect of tillage system, irrigation scheduling and depth on growth, yield attribute, yield and water use in wheat (Triticum aestivum). International Journal of Tropical Agriculture 2014 Vol. 32 No. 1/2pp. 51254

\section{How to cite this article:}

Vineet Kumar, R.K. Naresh, Vipin Kumar Sagar and Praveen Kumar Sagar. 2018. Performance of Wheat (Triticum aestivum L.) under Tillage Practices and Water Regime on Water Productivity and Economics. Int.J.Curr.Microbiol.App.Sci. 7(03): 3072-3081. doi: https://doi.org/10.20546/ijcmas.2018.703.357 